\title{
Stockage de déchets à vie longue en formation géologique profonde : limitation des circulations par barrières hydrauliques perméables
}

\author{
par Marc Boisson et Eric David
}

du LHF, Laboratoire d'Hydraulique de France

\author{
par Luc Chaudon et Serge Richard
}

du CEA, Centre d'Etude de la Vallée du Rhône, Marcoule

\section{INTRODUCTION}

Depuis plusieurs années le CEA a développé une collaboration avec le Laboratoire d'Hydraulique de France pour étudier les aspects liés à l'hydraulique dans le champ proche de stockage en formation géologique profonde de déchets radioactifs de type $C$ : propagation de radioéléments, effets thermo-hydrauliques, interaction entre la structure de stockage et le champ hydraulique proche,...

Le principe général guidant actuellement la recherche de concepts de stockage en formation géologique profonde repose sur le concept «multibarrière » [1] qui consiste à interposer plusieurs barrières de confinement, les moins perméables possibles, entre les radioéléments et la biosphère: conditionnement des déchets, barrière ouvragée et barrière géologique.
Indépendamment de la caractérisation de la barrière géologique, une difficulté importante consiste à mettre en place des matériaux de scellement (destinés à être disposés entre le colis et le massif hôte) dont la perméabilité se rapproche de celle du massif non dégradé et dont les caractéristiques physico-chimiques soient stables dans le temps (notamment durant la phase thermique où l'échauffement dû aux colis peut provoquer des micro-fissures). Une autre difficulté réside dans l'impossibilité d'assurer parfaitement le scellement hydraulique de la zone dégradée des roches à proximité des parois.

L'approche proposée ici, complémentaire de l'approche précédente, consiste à concevoir un concept de stockage avec des puits drains à l'amont et à l'aval hydraulique de façon à constituer une barrière hydraulique drainante qui isole la zone de stockage proprement dite.

The general principle which is at present followed for the concepts of deep storage of long-lived radioactive waste aims to separate the radioelements from the biosphere with isolation barriers, using the miltibarrier concept: Waste conditioning, worked bartier and geological barnier The original approach which is presented thereafter must be considered as complementary to the previous one. It consists in creating a storage concept with a hydraulic draining bantier which would be located in the close field, in order to isolate the actual storage area hydraulically, and so to form a partial hydraulic Faraday cage.

A detailed tridimensional nodel, which was carried out with the help of DEDALE-3D software (hvdraulic and transportation calculation), enabled the researchers to analuse the runoff in the close field in a reference site (wilhout any draiming barrier), which is particularly characterized by important contrasts in geonetry and permeability. The analysis has guided the research for storage concepts which seem to have an increased containment capacity. Then, the tridimensional modeling of these concepts enabled the researchers to prove the retevance of the followed approach. 
Dans le présent article, on s'intéresse, dans un premier temps, à l'analyse du champ hydraulique autour de la zone de stockage au moyen d'un modèle tridimensionnel détaillé d'un concept de référence. L'objectif initial était de vérifier en particulier si, a posteriori, une approche simplifiée (écoulements supposés bidimensionnels, homogénéisation des perméabilités de la zone de stockage) pouvait être acceptable. Les différentes analyses antérieures montrent en effet qu'aucune simplification ne permet de reproduire correctement l'hydrodynamique locale.

Dans un deuxième temps, on montre que l'analyse détaillée du champ hydraulique, en particulier des flux issus des puits de stockage, permet d'orienter la recherche de concepts limitant la propagation des radioéléments dans une direction nouvelle.

\section{Analyse tridimensionnelle détaillée des écoulements dans le champ proche d'un concept de stockage de référérence}

\subsection{Géométrie du concept de stockage de référence}

Le concept de stockage considéré est constitué d'un ensemble de puits verticaux dans lesquels sont entreposés des colis de déchets radioactifs de type C. Ces déchets se caractérisent par une vie longue et un fort dégagement thermique dû à l'auto-absorption des rayonnements béta et gamma [1]. La profondeur des puits ainsi que leur espacement sont déterminés en partie d'après des considérations liées à la charge thermique du stockage. Les puits de stockage sont connectés à une galerie de manutention, chaque module de stockage ainsi constitué étant relié aux autres modules par l'intermédiaire d'une galerie de service (fig. l).

\subsection{Modélisation du concept de stockage de référence}

Le modèle conceptuel déduit du concept de stockage de référence et servant de base au modèle mathématique, correspond à un site générique (fig. 2)). Le massif est considéré comme un milieu poreux homogène isotrope et la loi de Darcy est supposée applicable, l'intention ici n'étant pas de prendre en compte toute la complexité de la barrière géologique mais d'insister sur les caractéristiques géométriques du stockage. La perméabilité du massif $\left(10^{-11} \mathrm{~m} / \mathrm{s}\right)$ correspond aux valeurs couramment envisagées pour un stockage en formation géologique profonde. Les galeries et les puits sont remplis par un matériau de perméabilité supérieure à celle du massif $\left(10^{-6} \mathrm{~m} / \mathrm{s}\right)$. La galerie de service est supposée avoir une influence hydraulique mineure et n'a pas été prise en compte. Le système hydraulique est délimité par deux discontinuités majeures (sans perte de charge sur la totalité de leur hauteur) situées verticalement de part et d'autre du stockage, le gradient hydraulique régional étant de $1,5 \%$. Le milieu est supposé saturé en eau et les effets thermohydrauliques sont considérés comme négligeables [2]. Pour des raisons de symétrie, seulement la moitié d'un module de stockage est considérée dans le modèle mathématique.

\subsection{Modélisation numérique et analyse du champ hydraulique proche}

Un modèle mathématique a ensuite été construit avec le système de modélisation DEDALE-3D $[3,4,5]$ en prenant en

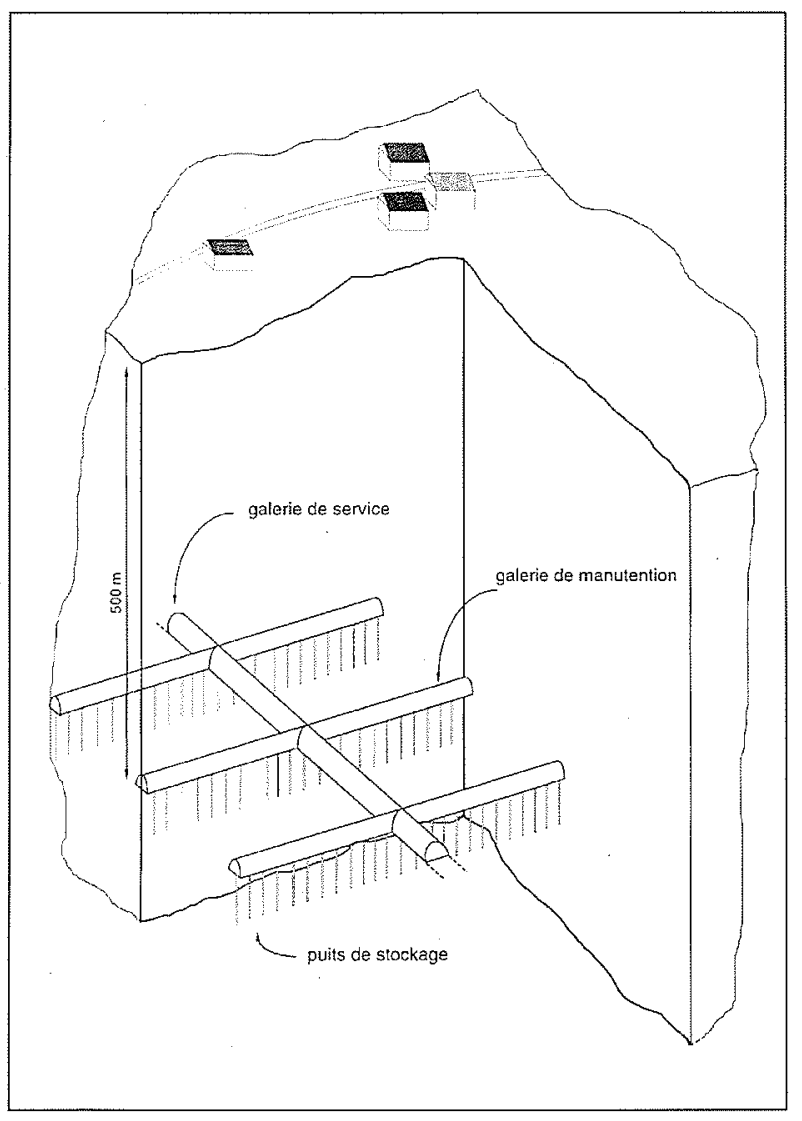

1. Stockage profond avec puits verticaux.

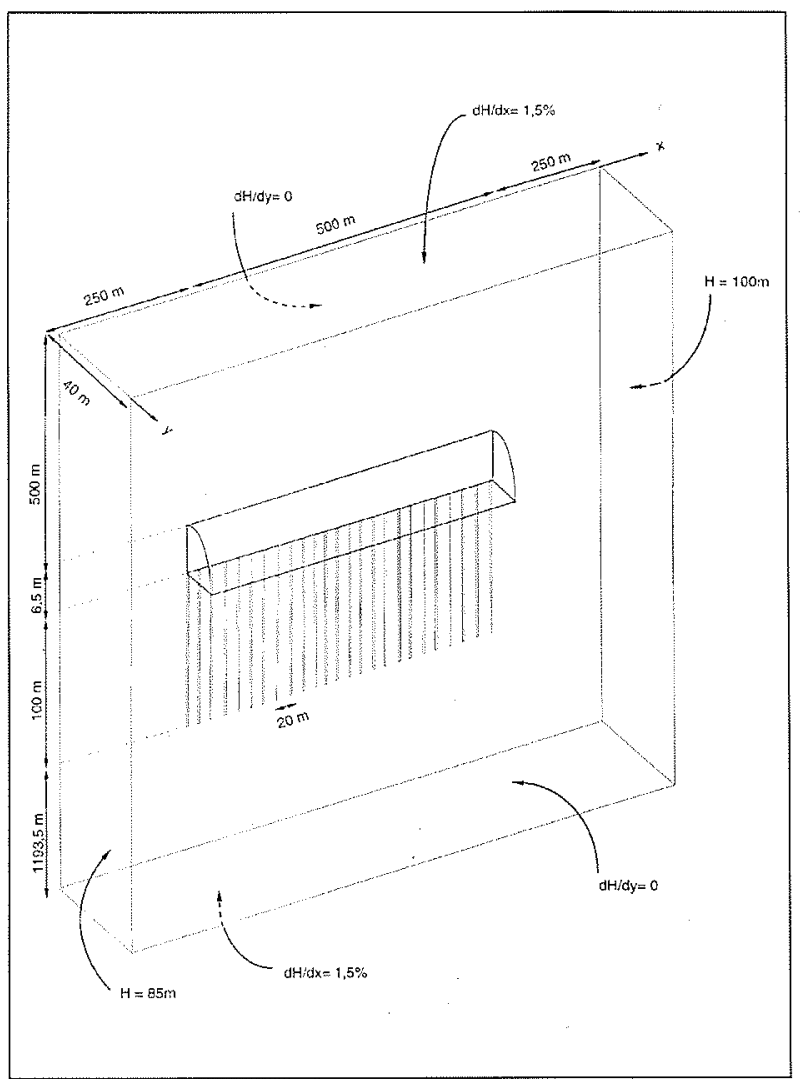

2. Modèle conceptuel du concept de stockage de référence. 


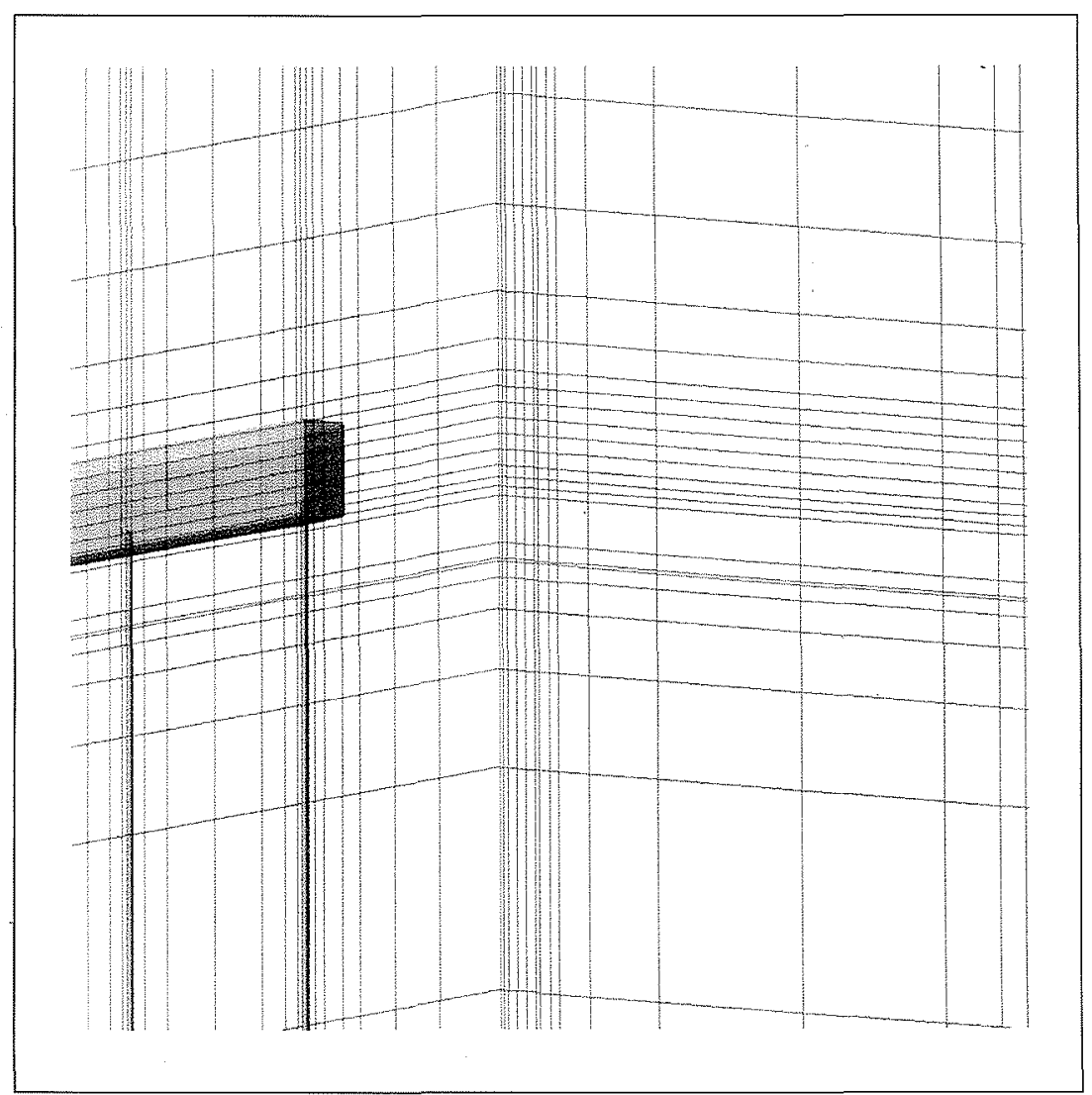

3. Vue détaillée du maillage 3D représentée par 2 plans de coupe (selon $\mathrm{OxOz}$ \& $\mathrm{OyOz}$ ).

compte la structure détaillée du stockage puisque chaque puits de stockage est représenté (fig. 3) et que la section transversale de la galerie est représentée par un grand nombre de mailles. Le modèle ainsi construit comporte environ 360000 mailles.

Le système de modélisation DEDALE-3D permet de modéliser les écoulements et le transport en zone saturée et en zone non saturée, de prendre en compte les hétérogénéités et les anisotropies des sols, des singularités hydrauliques ainsi que l'influence des variations de la densité sur l'écoulement, mais c'est surtout la robustesse des algorithmes utilisés et les possibilités de contrôler les bilans de flux localement qui ont été mises à profit au cours de ces travaux. En effet, les contrastes de perméabilité et les fortes hétérogénéités de géométrie rencontrées auraient pu engendrer des difficultés vis-à-vis de la convergence des calculs.

Dans le but de garantir la fiabilité des résultats obtenus plusieurs volumes de contrôle ont été introduits dans le modèle notamment un volume de contrôle global et un volume de contrôle local englobant la partie amont de la galerie de manutention ainsi que les 3 premiers puits de stockage. Durant la procédure de calcul, alors que les écarts de charges devenaient minimes et que le bilan global était équilibré le bilan local ne l'était pas encore. Dans ce second volume de contrôle les écarts de charge sont les plus notables et un état de convergence acceptable est plus difficile à obtenir.

C'est uniquement lorsque l'ensemble des bilans (global et locaux) a été équilibré qu'une analyse détaillée du champ hydraulique et des flux, en particulier ceux issus des puits de stockage, a pu être entreprise.

La compréhension des écoulements autour de la zone de stockage est facilitée par la visualisation tridimensionnelle des lignes de courant et l'analyse locale des flux au moyen de surfaces de contrôle.

Des lignes de courant ont été tracées à partir de l'amont du modele (fig. 4). Certaines illustrent bien la nature tridimensionnelle des écoulements aux alentours de la zone de stockage. Ainsi des lignes de courant sub-horizontales sont présentes dans le massif à proximité des puits de stockage. A l'amont du stockage un grand nombre de lignes de courant atteignent les puits de stockage non pas uniquement à la base de ceux-ci mais sur toute leur hauteur et selon une direction non parallèle au plan de l'écoulement régional (fig. 5).

Les éléments qui viennent d'être mentionnés ainsi que la représentation des isocharges (fig. 6) mettent en évidence la réalité d'écoulements tridimensionnels dans le champ proche et justifient la mise en place d'un modèle tridimensionnel détaillé par rapport à une approche simplifiée (i.e. modèle tridimensionnel avec homogénéisation des perméabilités de la zone de stockage ou modèle bidimensionnel avec ou sans homogénéisation).

La contribution des principaux éléments de la structure de stockage a été précisée grâce à la possibilité d’introduire des surfaces de calcul de flux (fig. 7).

L'importance des flux latéraux dans la troisième direction y (fig. 2), i.e. perpendiculaire au plan de l'écoulement régional, est notable, ils correspondent à plus des trois quarts des flux atteignant la zone de stockage.

Par ailleurs, environ $65 \%$ du flux à mi-galerie est issu des puits de stockage, de plus une hétérogénéité des contributions de chaque puits est observée puisque le premier puits (le plus à l'amont) représente $20,5 \%$ du flux à migalerie et les 4 premiers puits $46,5 \%$. 


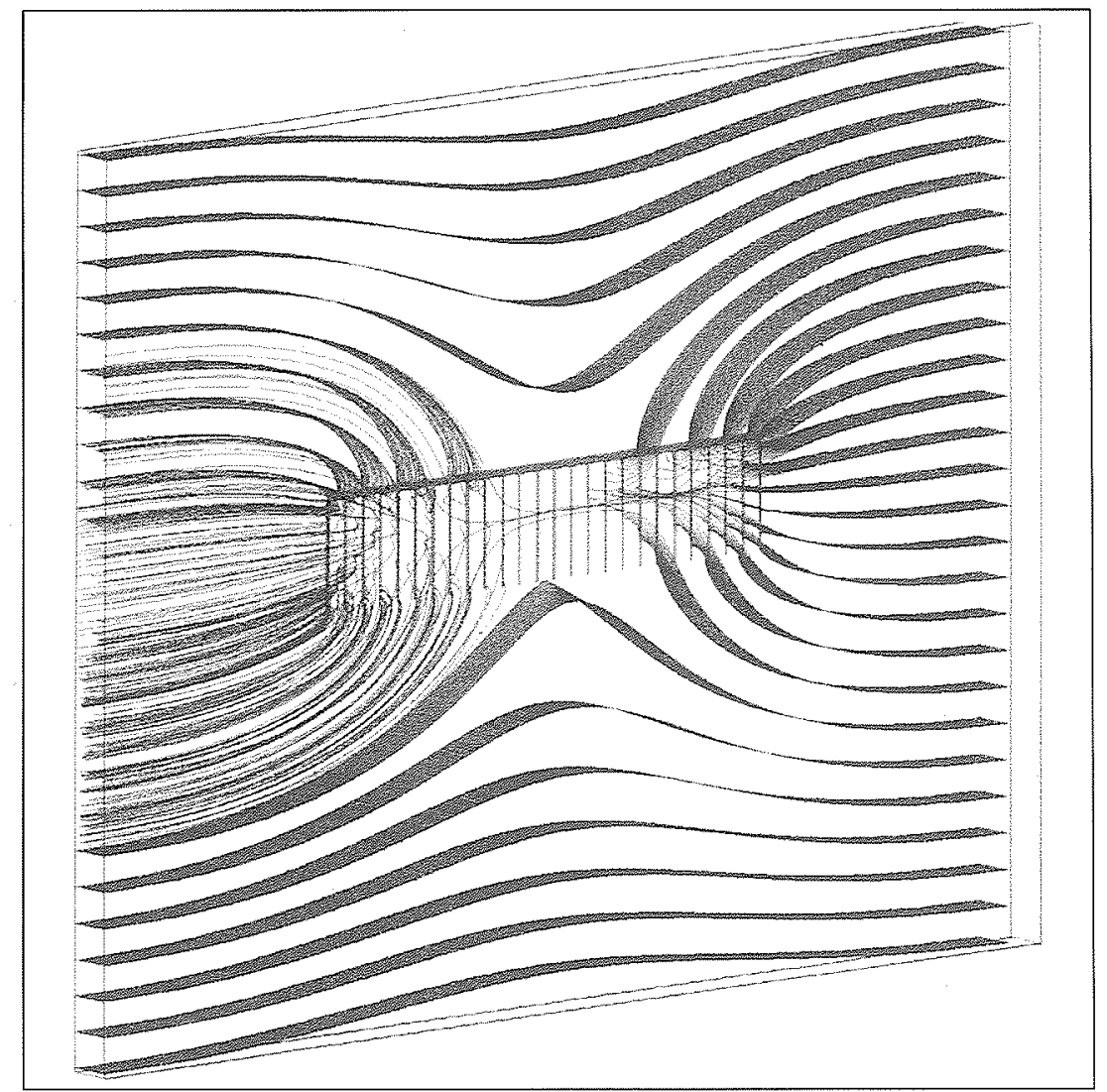

4. Concept de référence : lignes de courant dans le champ proche.

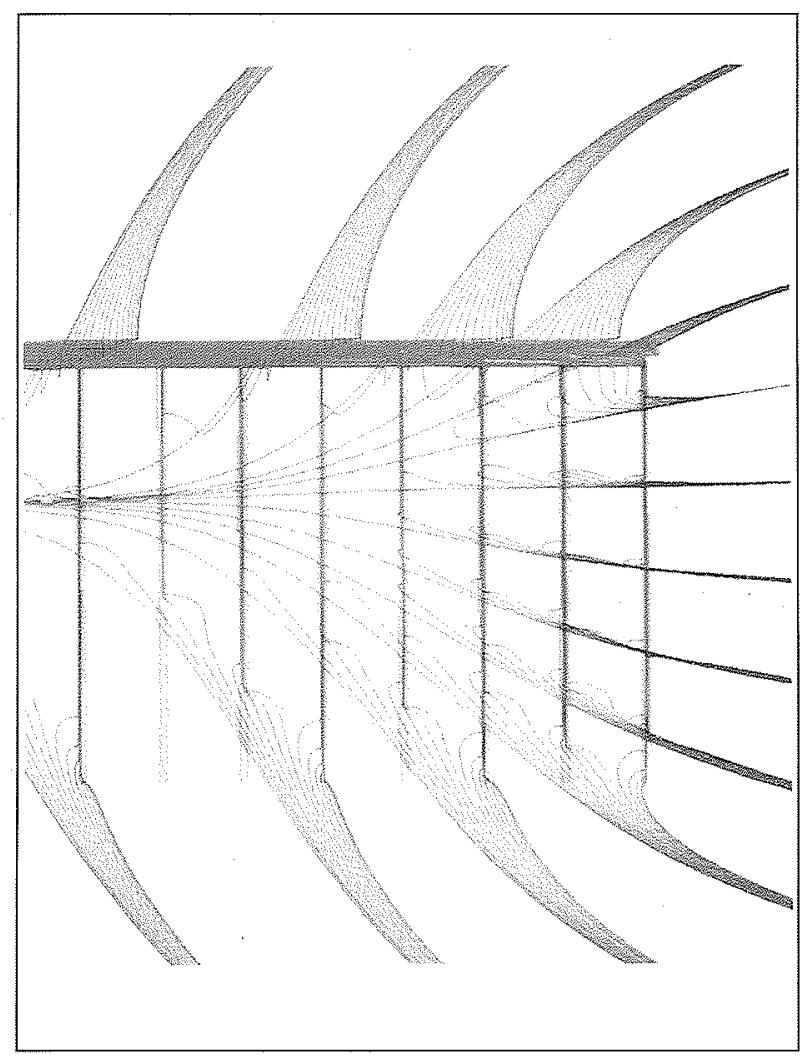

5. Concept de référence : lignes de courant à l'amont du stockage.

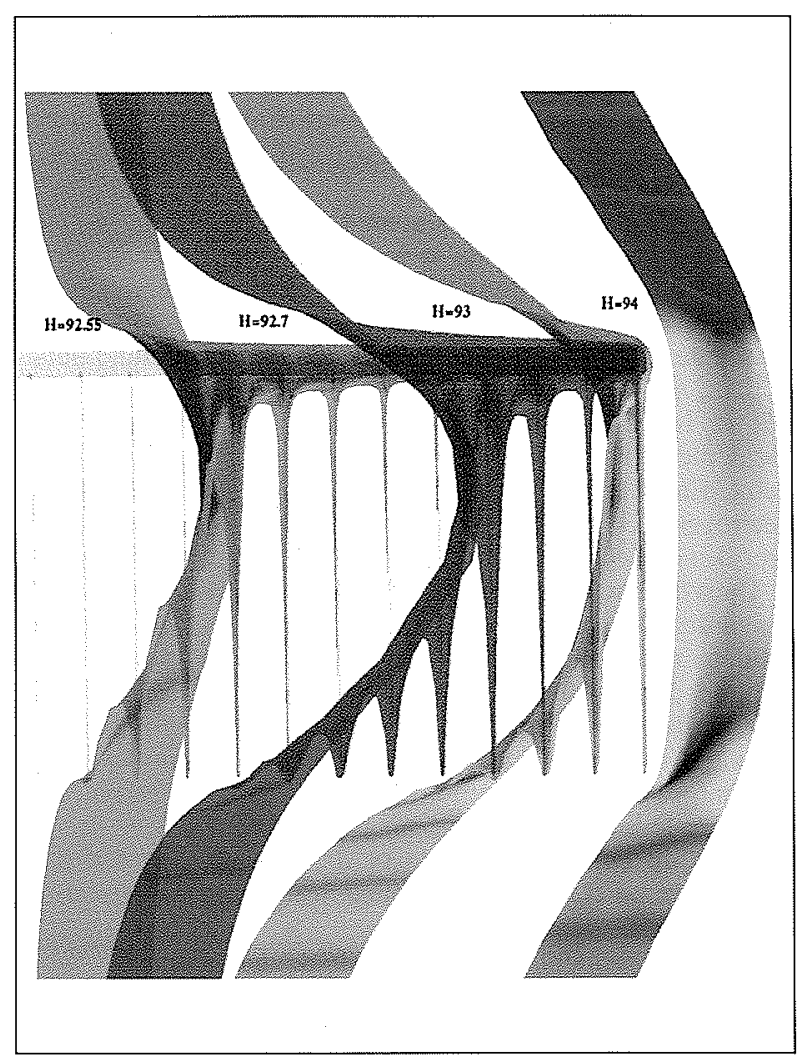

6. Concept de référence : isocharges à l'amont du stockage. 


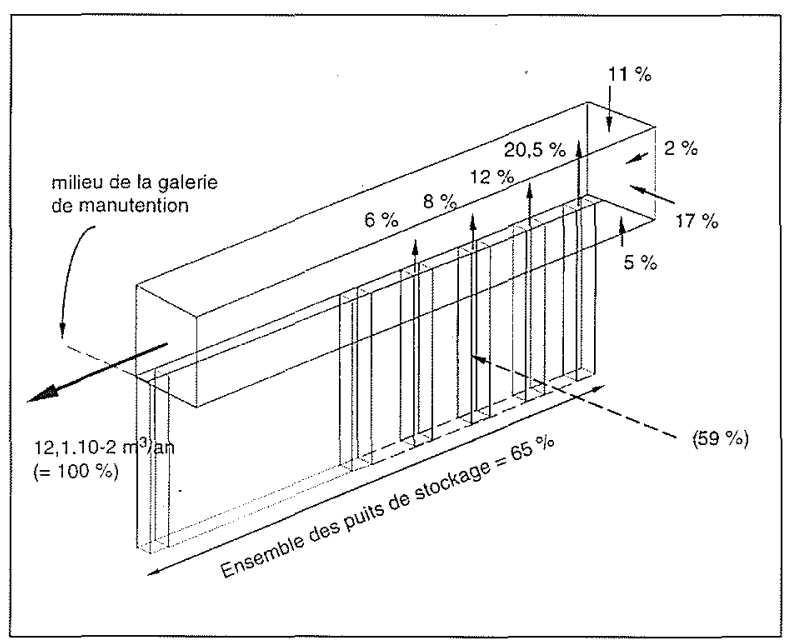

7. Concept de référence : répartition locale des flux.

L'importance des flux issus des puits de stockage s'explique par leur comnexion à la galerie de manutention qui les relie ainsi à l'aval hydraulique de la zone de stockage, créant de cette façon un cheminement préférentiel pour l'écoulement régional.

\section{Amélioration de la capacité de confine- ment par barrières hydrauliques per- méables}

L'influence d'une telle structure, plus perméable que le massif hôte, sur les écoulements est alors amplifiée pour concevoir des concepts de stockage présentant une capacité de confinement accrue,
Plusieurs modèles ont été construits avec des puits drains (sans colis) reliés entre eux par des galeries et situés à l'amont et à l'aval hydraulique des puits de stockage. L'étude de ces différents modèles a montré qu'avec un nombre restreint de puits drains entre deux galeries de manutention voisines, une efficacité proche de celle d'un "mur drainant "était obtenue. Une cage de Faraday hydraulique partielle est ainsi constituée en isolant hydrauliquement la zone de stockage qu'elle englobe (les radioéléments sont ainsi privés de vecteur pour leur propagation ( ${ }^{1}$ ).

\subsection{Exemple d'un stockage avec puits drains}

La sensibilité de la capacité de confinement vis-à-vis du nombre de puits drains, de leur profondeur, de la profondeur des puits de stockage et de la distance entre les galeries de manutention (tout en respectant les contraintes liées à la charge thermique du stockage) a été examinée et le concept présenté ci-dessous (fig. 8) illustre la pertinence de l'approche suivie.

\subsection{Analyse hydraulique du champ proche}

De la même façon que pour le concept nominal des lignes de courant ont été tracées à partir de l'amont du modèle (fig. 9 et 10 ).

La plupart des lignes de courant qui auparavant atteignaient les puits de stockage sont maintenant captées par les puits drains.

Le rôle des puits drains est confirmé par le calcul des flux localement (fig. $/ 1)$ : la zone de stockage ne représente plus qu'environ $3 \%$ du flux à mi-galerie, ce qui correspond à une

(1) Par convection

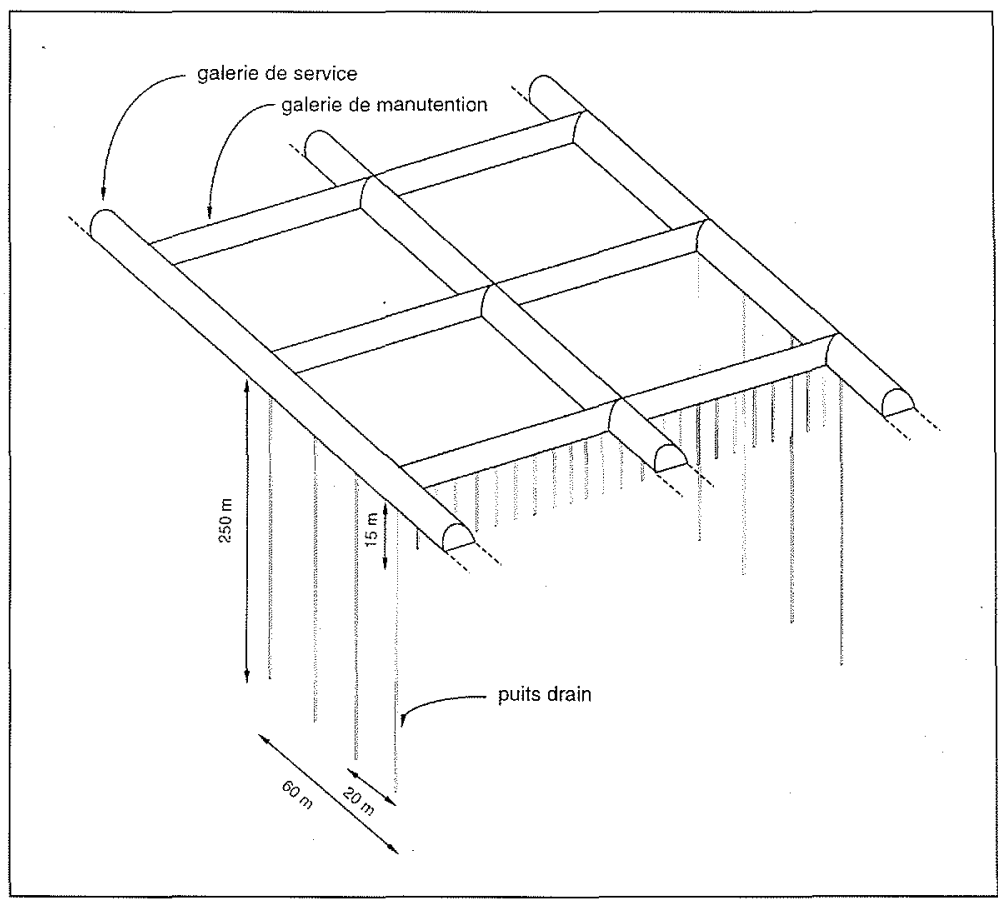

8. Géométrie d'un concept avec puits drains. 


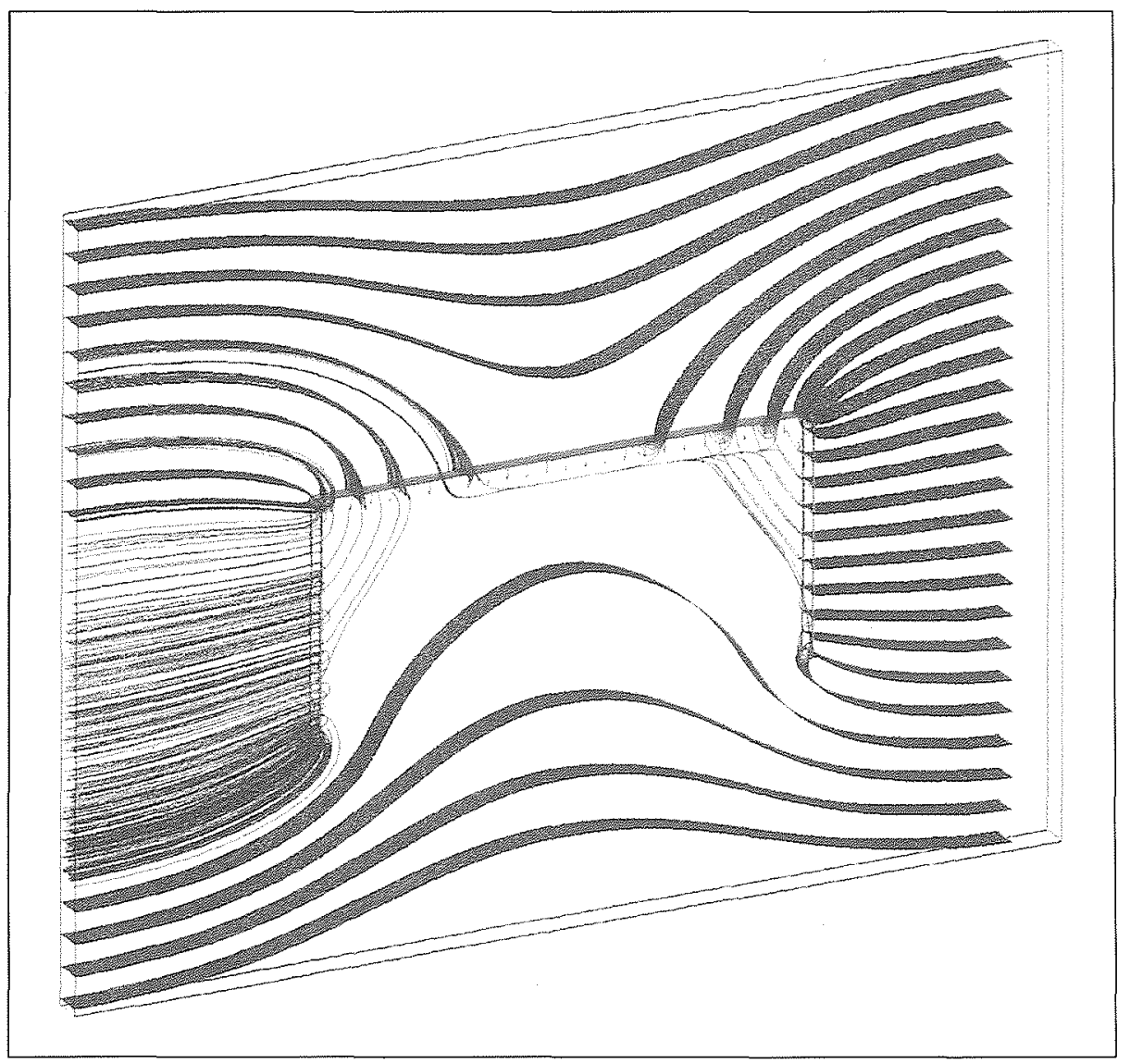

9. Concept avec puits drains : lignes de courant dans le champ proche.

réduction de $95 \%$ par rapport à la configuration de référence. Ces résultats mettent en évidence l'efficacité d'une cage de Faraday hydraulique partielle. Il apparaît possible qu'une telle cage (partielle, voire complète ?) soit opérationnelle d'une part, dans le cas d'un changement du gradient hydraulique régional et d'autre part, dans le cas d'autres concepts, ceux par exemple comportant des alvéoles de stockage horizontales.

\section{CONCLUSION}

Un modèle tridimensionnel détaillé du champ proche d'un stockage a permis, non seulement de mieux comprendre les interactions entre la structure de ce stockage et les écoulements au voisinnage proche, mais aussi d'orienter la recherche de concepts de stockage présentant a priori une capacité de confinement améliorée et de le vérifier a posteriort.

Le principe de l'isolement hydraulique par barrière hydraulique perméable peut être extrapolé à d'autres contextes que celui des stockages de déchets radioactifs en formation géologique profonde, notamment au cas de sites industriels de surface (isolement par rapport à la contamination que pourrait provoquer la zone de stockage mais également isolement d'une zone sensible vis-à-vis d'une contamination externe). C'est dans ce sens et à la suite des présents travaux que le CEA a pris ses dispositions de propriété industrielle.

\section{Remerciements}

Nous tenons à remercier la société AVS-UNIRAS qui a mis à notre disposition le logiciel AVS de visualisation tridimensionnelle des écoulements.

\section{Références}

[1] Hoorelbeke J.M. (1993). - "Stockage profond de déchets radioactifs et réversibilité, d'après la loi du 30 décembre 1991 \%. Geoconfine 1993. Arnould, Barrès \& Cômes (Ed). Balkema, Roterdam.

[2] Cordier E., Goblet P. (1987). - "Effet thermique et hydraulique d'un stockage de déchets radioactifs dans l'argile de Boom », Report LHM/RD 87/58.

[3] Usseglio-Polatera J.M., aboviaddé A., Molinaro P. et RANGOGNI R - 3-D Modelling of coupled groundwater flow within saturated and unsaturated zones". VIII Intern. Conf. in Computational Methods in Water Resources. Venise, Italie, 11-15 juin 1990.

14] Usseglio-Polatera J.M., Jardin P., Molinaro P. "DEDALE: un système de modélisation pour la gestion des aquifères côtiers ». XXI Journées de l'Hydraulique. Sophia Antipolis, 29-31 janvier 1991.

[5] Abolisaudé A., Jardin P., Pelmont F. (1993). "DEDALE: un ounil d'analyse des risques induits par le stockage de déchets ", Geoconfine 1993, Arnould, Barrès \& Cômes (eds). Balkema, Rotterdam. 


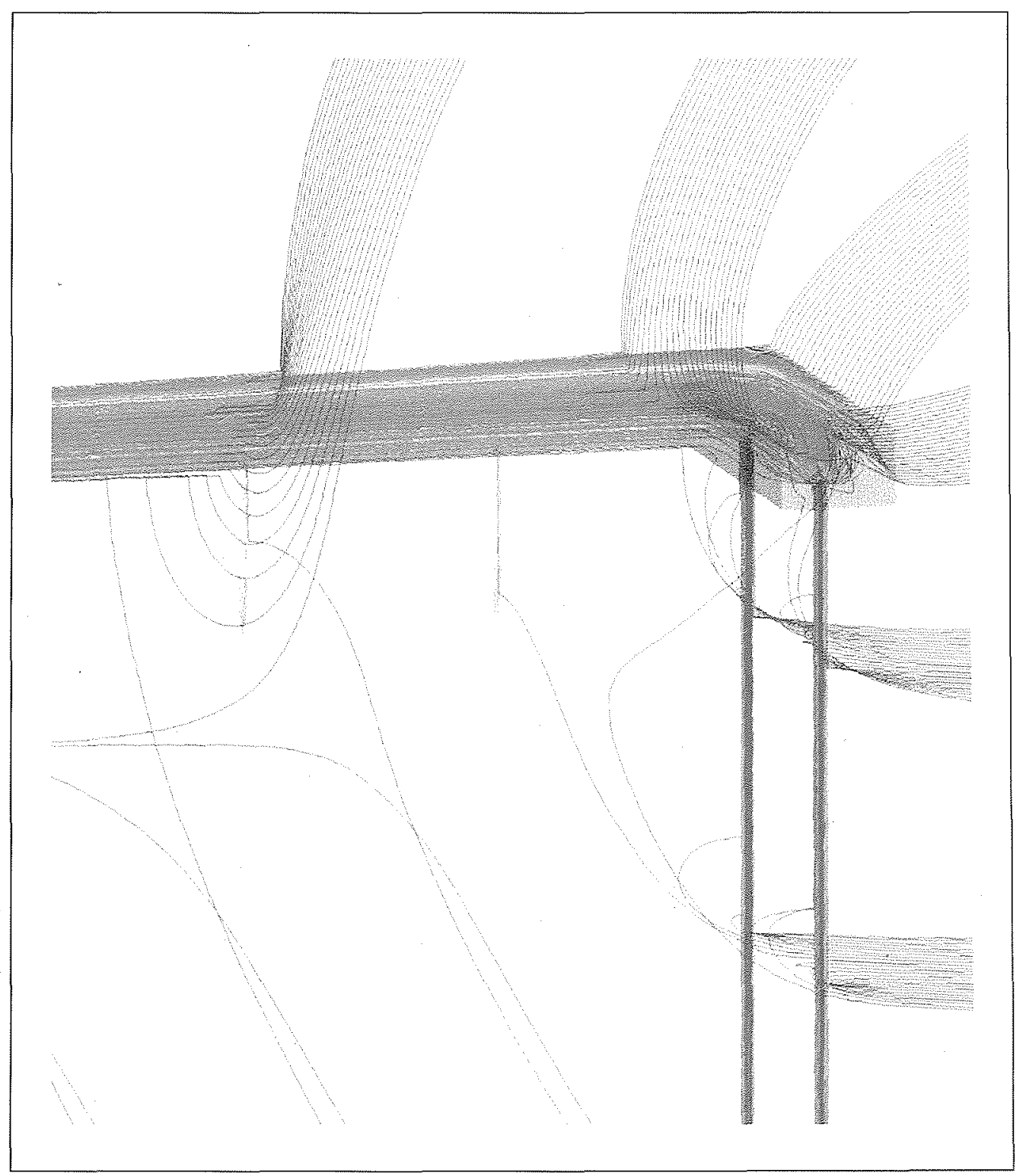

10. Concept avec puits drains : lignes de courant à l'amont du stockage.

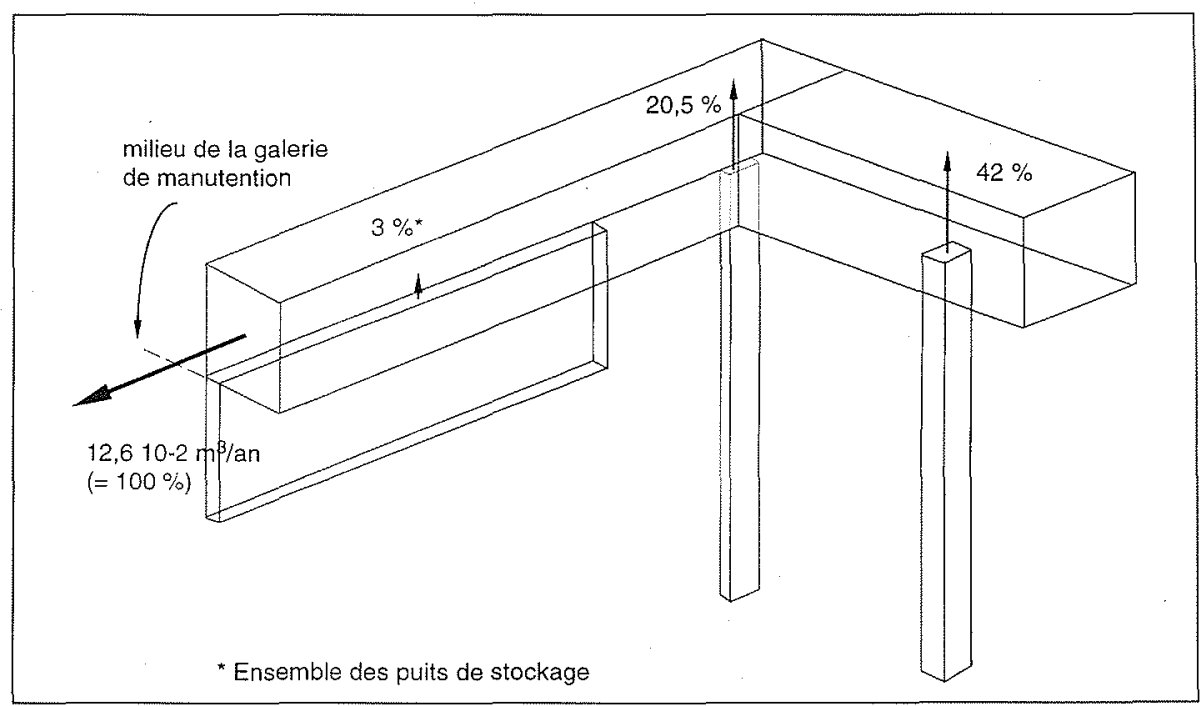

11. Concept avec puits drains : répartition locale des flux. 